\title{
COVID-19 and Cardiovascular Disease: a Global Perspective
}

\author{
Alessandra Pina ${ }^{1} \cdot$ Silvia Castelletti ${ }^{2}$ iD \\ Accepted: 29 April 2021 / Published online: 19 August 2021 \\ (C) The Author(s), under exclusive licence to Springer Science+Business Media, LLC, part of Springer Nature 2021
}

\begin{abstract}
Purpose of Review This review aims to assess the global impact of the COVID-19 pandemic on the cardiovascular diseases (CVDs), trying to assess the possible future trajectory of the CVDs and their management.

Recent Findings The COVID-19 pandemic has had a deleterious impact on the CV risk factors, with an increase in both sedentary and unhealthy food habits. The fear of contagion has decreased the access to the emergency systems with an increase in out-of-hospital-cardiac-arrests and late presentation of acute myocardial infarctions. The closure of the non-urgent services has delayed cardiac rehabilitation programmes and chronic clinical care.

Summary As a result of the COVID-19 pandemic impact on the population habits and on the management of CVDs, we will probably face an increase in CVD and heart failure cases. It is crucial to use all the non-traditional approaches, such as telemonitoring systems, in order to overcome the difficulties raised by the pandemic.
\end{abstract}

Keywords COVID-19 - Coronavirus · Cardiovascular disease · Telemonitoring · e-Health · Primary prevention · Cardiac rehabilitation · Diet · Physical activity · Out-of-hospital-cardiac-arrest

$\begin{array}{ll}\text { Abbreviations } & \\ \text { AHA } & \text { American Heart Association } \\ \text { AHF } & \text { Acute heart failure } \\ \text { AMI } & \text { Acute myocardial infarction } \\ \text { COVID-19 } & \text { Coronavirus disease 2019 } \\ \text { CVD } & \text { Cardiovascular disease } \\ \text { EMS } & \text { Emergency medical system } \\ \text { ESC } & \text { European Society of Cardiology } \\ \text { GAPPA } & \text { Global Action Plan on Physical Activity } \\ \text { OHCA } & \text { Out-of-hospital-cardiac-arrest }\end{array}$

This article is part of the Topical Collection on Global Cardiovascular Health

Silvia Castelletti

s.castelletti@auxologico.it

Alessandra Pina

alessandra.pina2@gmail.com

1 IRCCS Istituto Auxologico Italiano, Department of Cardiovascular, Neural and Metabolic Sciences, piazzale Brescia 20, 20149 Milan, Italy

2 IRCCS Istituto Auxologico Italiano, Cardiomyopathy Unit and Center for the Cardiac Arrhythmias of Genetic Origin, Department of Cardiovascular, Neural and Metabolic Sciences, piazzale Brescia 20, 20149 Milan, Italy
SARS-CoV-2 Severe acute respiratory syndrome coronavirus-2

"Life isn't about waiting for the storm to pass... It's about learning to dance in the rain." Vivian Greene

\section{Introduction}

It has already been more than one year since the novel coronavirus disease 2019 (COVID-19), officially known as SARS-CoV-2 (severe acute respiratory syndrome coronavirus-2), pandemic has started [1], and surely it has become a remarkable challenge for healthcare systems around the world.

The tragic consequences of the infection in terms of deaths and intensive care units admissions are evident to everyone. However, the COVID-19 effects are not limited to those directly linked to the infection. The pandemic has also had major repercussions on the lifestyle of everyone, on the healthcare systems in general, and on the non-COVID patients too, who have not been able to receive the same level of assistance as before. 
The rapid increase of cases and the fear of being infected during a hospital staying have led to a progressive reduction in emergency department attendances, even for acute coronary syndromes, which contributed to the increase in out-of-hospitalcardiac-arrest (OHCA) [2]. The delayed access to the treatments will lead to an increase in infarction-related complications. Progressive lockdown measures delayed most elective procedures, outpatient visits, and rehabilitation programmes with effects on the most vulnerable cardiovascular patients [3]. The restrictions to contain the infection have caused lifestyle changes in a deleterious direction, with a possible impact on the future prevalence of cardiovascular diseases [4]. All the indirect consequences of the COVID-19 pandemic not only have changed our daily practice, but will have a long-term impact. Cardiologists need to be prepared for a different cardiovascular disease scenario in the future and take advantage of this challenges time implementing the current healthcare modalities with new ehealth methods $[5 \bullet, 6]$ (Fig. 1).

\section{The Impact on Primary Cardiovascular Prevention Risk Factors}

Regular physical activity is considered an important component for primary and secondary prevention of cardiovascular disease (CVD), reducing cardiovascular and all-cause mortality. According to the recently published Guidelines on Sports Cardiology and Exercise in patients with cardiovascular disease of the European Society of Cardiology [7••] (ESC), moderate exercise should be performed on most days of the week, amounting to a minimum of $150 \mathrm{~min} / \mathrm{week}$. The COVID-19 pandemic has forced the world to an unforeseen stop: the measures taken by the governments to contain the infection have involved the shutdown of all the recreational activities, including sports clubs and sports events, and the mandatory homestay. Considering that inactive individuals have about a two-fold higher relative risk of coronary artery disease in comparison with physically active people and taking in account all the beneficial effects of physical activity on metabolic disorders, immune system, mental health, and several non-cardiovascular diseases [8], the impact of this forced lockdown may be deleterious.

Between March and April 2020, a reduction of physical activity by $48 \%$ in 7-day rolling step count has been reported in the USA [9]. A reduction of physical activity was also reported by $49 \%$ of 1491 Australian adults in April 2020 [10, 11]; by $43 \%$ of 2381 Polish adults in April and May 2020 [12]; and by $41 \%$ of English individuals in April 2020 [13], in contrast with an increased physical activity reported by $21 \%, 19 \%$, and $31 \%$ of adults, respectively. Using continuous monitoring of daily step counts, Ding et al. reported a physical activity reduction in a cohort of 815 Chinese adults living in Shangai over 202 days and 164,630 persondays across different phases of the COVID-19 pandemic [14]. They found a sharp decline in daily step counts upon the lockdown by an average of 3796 steps, followed by a trend in 34-step per day increase until the end of the lockdown, which continued into the reopening phase at a slower rate of

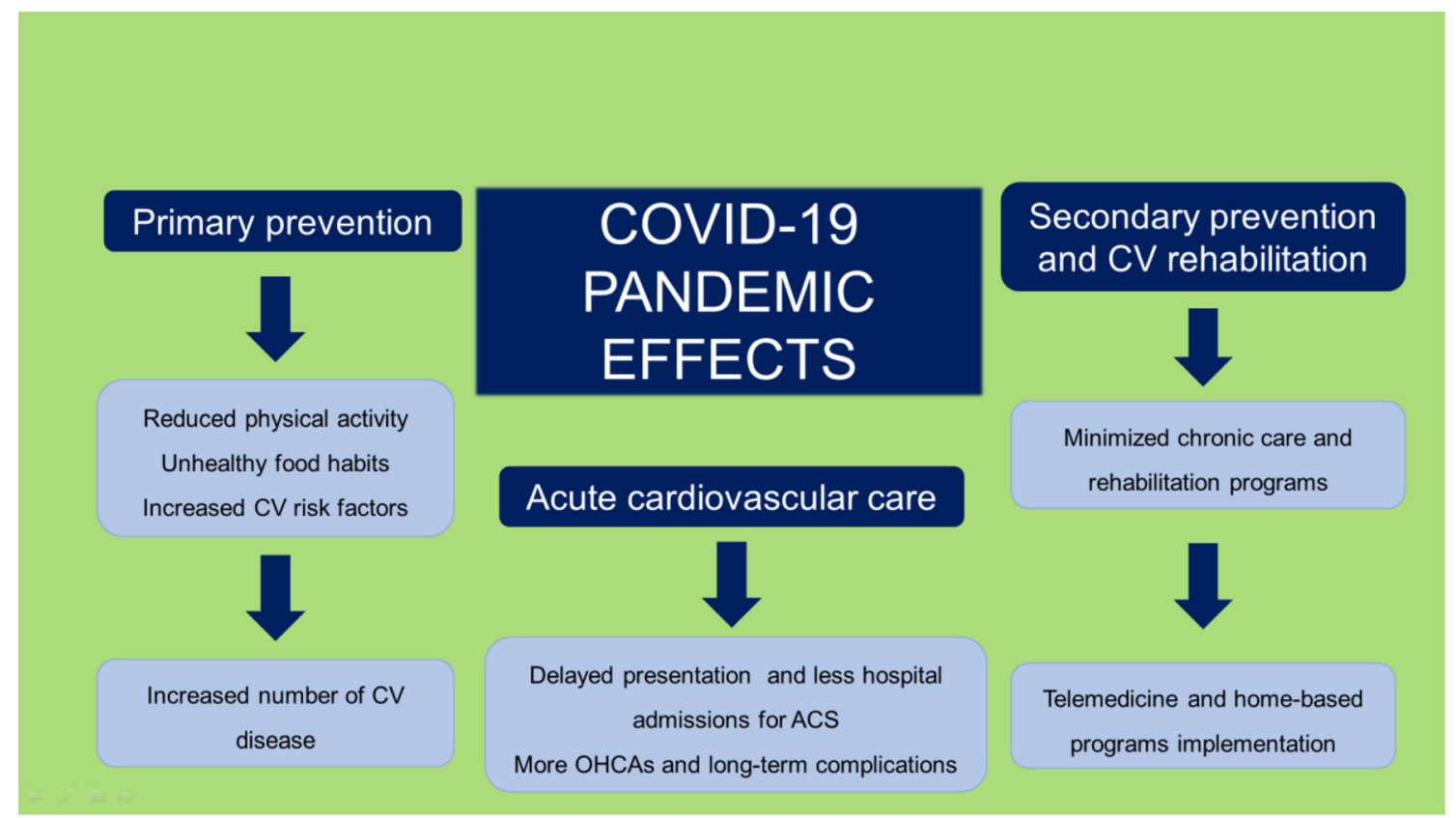

Fig. 1 Effects of the COVID-19 pandemic on cardiovascular diseases. CV, cardiovascular; OHCAs, out-of-hospital-cardiac-arrests; ACS, acute coronary syndromes 
5 steps per day [14]. Also, the workers who telecommuted in the Tokyo metropolitan area were less physically active and had longer sedentary behaviours [15].

Similar observations have also been reported in children and teenagers, not only in adults. From parents reports of 211 children of 35 US states, it has been registered a greater sedentary behaviour and a reduction in physical activity among children aged 5-13 years old [16]. A German study reports a decline in sports activities and an increase in recreational screen time among 1711 children aged 4-17 years old [17]. Barkely et al. compared the physical activity and sedentary behaviour in a sample of university students and employees pre and post the campus closure: they observed an increase in sedentary behaviour and a decrease in physical activity, the latter one being greater among the participants who were the most active before the pandemic [18].

The effects of the restrictions on physical activity, indeed, are not similar in all individuals and in all countries. Ding et al. report an attenuated increase in steps during the lockdown in older, married, university educated, insufficiently active at baseline, and with a "at-risk" body composition individuals [14]. A Canadian online survey indicates that $40.5 \%$ of inactive individuals became less active, while only $22.4 \%$ of active individuals decreased their active lev$\mathrm{el}$; on the other hand, $33 \%$ of inactive subjects and $40.3 \%$ of active individuals, respectively, became more active [19]. A Belgian survey registered a general increase in exercise frequencies as well as in sedentary behaviour: baseline low active subjects aged less than 55 years old started to exercise more during the lockdown; among the baseline active people, those older, with lower education and who used to exercise with friends or in a sports club, reduced their physical activity [20]. In a study on 3052 US adults from all 50 states, the subjects who met the physical activity guidelines prior to the restrictions decreased their physical activity by $32 \%$ on average [21]. This percentage reached a peak of $43 \%$ among the subjects in self-isolation, with an increase in sitting and screen time [21]. Also among the individuals who were not active before the restriction, those in selfisolation increased their sitting and screen time, while no significant change was reported in physical activity [21]. Italians living in north-western regions, instead, maintained a moderate amount of physical activity during the quarantine [22]. Also, among Swiss office workers, it was not observed a reduction in total physical activity during the first weeks of lockdown [23].

However, besides these differences, overall, the effect of the restrictions on physical activity has been deleterious worldwide. Using the data released by a step counter smartphone app, Tison et al. collected the data of 455,404 individuals from 187 different countries [24]. Worldwide, there was a $5.5 \%$ and a $27.3 \%$ decrease in mean daily steps within 10 and 30 days of pandemic, respectively [24].
These modifications in lifestyle are such a concern that a call to change the Global Action Plan on Physical Activity (GAPPA) 2018-2030 of the World Health Organization has been done, and probably the goals of the GAPPA will be revised [25].

Also, the diet is one of the milestones of a healthy body and mind [26], and food habits have changed during the COVID19 pandemic.

An international survey including 35 research organizations from Europe, North Africa, Western Asia, and America registered more unhealthy food consumption and meal patterns, including the type of food, snacks between meals, eating out of control, and number of main meals [27]. A review of 12 articles analysing the preliminary effects of the quarantine on dietary habits revealed a sharp rise of carbohydrates sources consumption, especially those with a high glycemic index, such as homemade pizza, as well as frequent snacks [28]. Both an Italian [29] and Spanish [30] study reported an increase adherence to the Mediterranean diet; however, increase consumption of "unhealthy" food was also observed among Spanish subjects [30]. A study including 700 Chilean subjects reports increase food consumption, with $51.3 \%$ of subjects declaring to eat more and eating junk food and fried foods more than before [31].

These changes are concerning given the well-known health risks associated with physical inactivity, unhealthy food habits, and the resulted gain of weight. They are even more concerning given that they may turn from short term to permanently fixed changes with exacerbations of cardiovascular diseases [4]. This is the reason for which video- or app-guided exercise training should be encouraged [6].

\section{The Impact on Acute Cardiac Care}

The coronavirus pandemic deeply and rapidly changed the population's approach to the health system and the way healthcare services are delivered [32]. The reduction in emergency departments' attendance was immediate, caused both by fear of contagion in a hospital setting and the willingness not to further overload the healthcare system [2, 32]. Both these reasons may explain some citizens' reluctance to activate the emergency medical system (EMS), which itself has been focused on managing the pandemic.

De Rosa et al. analysed acute myocardial infarction (AMI) admission rates during the COVID-19 outbreak compared to a control period in 2019 [33]. They found a 50\% reduction of AMI admissions across Italy, together with an increase in fatality and complication rates [33]. Interestingly, this trend was more significant for NSTEMI than for STEMI, suggesting as an addictive cause the need to postpone non-urgent procedures and the tendency to use more pharmacological reperfusion [33]. This tendency is confirmed in other 
countries, as seen in a study held at the Minneapolis Heart Institute that shows a $38 \%$ reduction in US catheterization laboratory activations [34], similar to a $40 \%$ reduction documented in Spain [35]. Also, the shortage of intensive care unit beds, full of COVID-19 patients, reduced the possibility to perform cardiac surgeries that needed to be rescheduled once the peak was reached.

Even though most studies focused on the impact on acute coronary syndrome admissions, hospitalizations for many other cardiovascular causes were affected as well. Recently, Bollmann et al. reported that in Germany hospitalizations also decreased for heart failure, arrhythmias, valvular heart disease, arterial hypertension, and peripheral vascular disease, reaching a nadir of over $40 \%$ reduction [36]. A similar study showed an important decline in hospitalization rates for acute heart failure (AHF) in London, with higher rates of more severe symptoms and NYHA class III/IV, suggesting that patients with milder clinical conditions have avoided presenting to the hospital [37].

One of the dramatic consequences is a parallel increase in out-of-hospital cardiac arrest (OHCA), documented in different studies. Baldi et al. analysed OHCA rates in 2020 compared to the same period in 2019 in Italy, and found a 58\% increase, strongly associated with the progressive outbreak of COVID-19 in the same region [38]. It was interesting to notice that the trend of OHCAs followed the shape of the epidemic curve, with a more rapid increase at the beginning and a following plateau phase [38]. Infection-related causes are likely to be present, such as the high probability of cardiac arrest due to hypoxia, one of the main causes of non-shockable rhythms. Nevertheless, pandemic-related causes must be taken into account. Together with the delay of activation of EMS by the patients, a 3-min median increase in EMS arrival was documented, explaining the prevalence of non-shockable rhythms upon arrival. Moreover, the need for isolation due to lockdown leads to a higher rate of unwitnessed cardiac arrests and a lower rate of bystander cardiopulmonary resuscitation. Characteristics of OHCAs were also different: in 2020, medical aetiology, home location, and not witnessed cardiac arrests were more frequent than in 2019 during the same period, together with worse outcomes in those patients who received an attempt of resuscitation by the EMS [38].

\section{The Importance of Cardiac Rehabilitation and Telemedicine Programmes}

To face the COVID-19 pandemic, many hospitals worldwide had to close non-urgent outpatients appointments, day cases, and also centre-based cardiovascular rehabilitation programmes. This has affected the vulnerable population $[3$, 39]. As such, it is crucial to assure appropriate chronic clinical care, as it is well established that delaying the start of cardiovascular rehabilitation may result in less improvement after a cardiac event. An exercise-based rehabilitation programme's cancellation after a myocardial infarction leads to a 20 to $30 \%$ increase in overall mortality and hospital admission rates after discharge [40]. The same problem regards people with heart failure that have a $40 \%$ higher risk of hospitalization if a proper rehabilitation programme is lacking [40, 41].

In fact, cardiac rehabilitation is a class IA recommendation by the American Heart Association (AHA) for secondary prevention after myocardial infarction, percutaneous coronary intervention, and coronary bypass graft surgery, and is also recommended in the setting of stable angina and chronic heart failure with reduced ejection fraction in order to reduce hospital admissions and mortality [42].

A widely proposed solution is to implement home-based programmes, where all interventions are delivered at patients' houses with the help of telemedicine tools [5•]. Even if an "athome" approach is still at its first steps, the ESC guidelines on cardiovascular diseases prevention state that "home-based rehabilitation with and without telemonitoring holds promise for increasing participation and supporting behavioural change" [43]. There are no definitive data about the long-term effectiveness of home-based programmes compared to centrebased ones, but the potential advantages of overcoming logistic barriers, especially during COVID-19 times, are evident [44].

A study comparing home-based cardiac rehabilitation, center-based rehabiliation and hybrid cardiac rehabilitation (a combination between center-based and home-based rehabilitation) shows that home-based cardiac rehabilitation can be a valid alternative program for patients with heart failure [45]. Also the AHA has concluded that home-based cardiac rehabilitation may be a reasonable option for selected patients who are eligible for rehabilitation programmes but cannot attend a traditional centre-based one [46].

In this setting, telemedicine plays a key role to ensure constant monitoring of patients' activity and safety during exercise. New tools such as physical activity trackers, mobile applications and wearable devices for heart rate and ECG allow a remote-controlled rehabilitation that clinicians can track to assure the same level of clinical care without the need to be face-to-face with the patients [46].

A recently published study demonstrated that an alternative home-based cardiac telerehabilitation model designed during the COVID-19 quarantine for people with coronary heart disease is able to increase cardiorespiratory fitness [47].

These alternative approaches can help the maintenance of rehabilitation programmes and the prevention of future increase in hospital readmissions and mortality rates, while avoiding a rise in COVID-19 transmission and a further burden of healthcare systems. Besides, the psychological advantage of a continuum of care must be considered in these 
peculiar times, as it can counterbalance the negative effect of social isolation.

As such, also the European Association of Preventive Cardiology has made a call for action for telerehabilitation in this difficult period [5•], a position paper to overcome challenges in digital health implementation in cardiovascular medicine has been published by the ESC e-Cardiology Working Group [48••], and several national associations have published consensus papers on how to deliver a cardiac rehabilitation programme during this period [49-52]. Also the European Association of Preventive Cardiology has timely provided general recommendations for cardiac rehabilitation activities during the COVID-19 pandemic [53].

\section{What Is in the Future?}

As the pandemic proceeds, there are growing concerns about the future of cardiology as we were used to know it. It is likely that the massive interruption of clinical outpatient consults, elective procedures, rehabilitation programmes, and clinical trials will have a significant impact on cardiovascular diseases prevalence and future everyday practice.

The combination of deleterious changes in lifestyle, reduced supply, and reduced demand for cardiovascular services will have a long-term impact on cardiovascular disease. Recently a study has estimated the direct incident impact of 31,205 and the prevalent impact of 62410 excess death in England and indirect effect of 49,932 to 99,865 deaths [54].

The population needs to be educated to seek medical care even during the COVID-19 emergency. The European Society of Cardiology has launched a campaign "you can't pause your heart" that urges anyone with symptoms of heart attack to seek care without delay. However, we are still facing a decrease in hospital admissions for acute cardiovascular issues. This is worrisome as it is unlikely that this decrease is due to a reduction of myocardial infarction rates. As it is more reasonable that the decrease in hospital admission is due to a lack of seeking of care, this will probably result in an increasing number of patients with post-myocardial infarct complications and heart failure.

In fact, for patients with acute myocardial infarction, the time frame between the onset of symptoms and the initial medical assessment is one of the most important predictors of mortality and severe complications [55]. Delayed presentation of AMI is associated with larger infarct size, increased mortality, and a higher risk of mechanical complications [56]. A study on myocardial infarctions in Hong Kong during the COVID-19 pandemic shows an average of 318 min between the infarction and the initiation of medical care, compared to a median of $82 \mathrm{~min}$ in the previous period [57]. The logic tragic consequence of a higher number of late diagnosis might be a superior incidence of post-ischemic heart failure that will need chronic care.

Missed or delayed diagnosis of acute cardiovascular disorders or rapid deterioration of chronic issues will result in a possible scenario of increased morbidity and mortality, for which we must be prepared.

Acknowledging the important consequences of less effective cardiologic care is important: although stay-at-home messages are needed to limit the contagions, it is also important to give precise information about the importance of urgent activation of emergency services to best protect the population. The media need to tell the entire population that seeking medical care is safe, that protection against the virus is always present in health centres, and that symptoms related to serious medical conditions need immediate attention.

Cardiologists need to be prepared for the worst. However, the implementation of the health system with remote monitoring and home-based programmes will surely represent a logistic advantage both for the patients and the caregivers. A time of crisis does not represent only a time of worry: it is also an opportunity, the opportunity to improve.

\section{Declarations}

Conflict of Interest The authors declare no competing interests.

Human and Animal Rights and Informed Consent This article does not contain any studies with human or animal subjects performed by any of the authors.

\section{References}

Papers of particular interest, published recently, have been highlighted as:

- Of importance

•. Of major importance

1. Zhou P, Yang XL, Wang XG, Hu B, Zhang L, Zhang W, et al. A pneumonia outbreak associated with a new coronavirus of probable bat origin. Nature. 2020;579:270-3.

2. Baldi E, Savastano S. Fear of contagion: one of the most devious enemies to fight during the COVID-19 pandemic. Disaster Med Public Health Prep. 2020:1-2. https://oi.org/10.1017/dmp.2020. 338.

3. Yeo TJ, Wang Y-TL, Low TT. Have a heart during the COVID-19 crisis: making the case for cardiac rehabilitation in the face of an ongoing pandemic. Eur J Prev Cardiol. 2020;27:903-5.

4. Mattioli AV, Sciomer S, Cocchi C, Maffei S, Gallina S. Quarantine during COVID-19 outbreak: changes in diet and physical activity increase the risk of cardiovascular disease. Nutr Metab Cardiovasc Dis NMCD. 2020;30:1409-17.

5. Scherrenberg M, et al. The future is now: a call for action for cardiac telerehabilitation in the COVID-19 pandemic from the secondary prevention and rehabilitation section of the European Association of Preventive Cardiology. Eur J Prev Cardiol. 2020: 
2047487320939671. https://doi.org/10.1177/2047487320939671. This paper highlights the importance of remote monitoring systems in secondary prevention and cardiac rehabilitation in the Covid-19 era, highlighting pro and cons of telemonitoring.

6. Lippi G, Henry BM, Sanchis-Gomar F. Physical inactivity and cardiovascular disease at the time of coronavirus disease 2019 (COVID-19). Eur J Prev Cardiol. 2020;27:906-8.

7.• Pelliccia A, et al. ESC Guidelines on sports cardiology and exercise in patients with cardiovascular disease. Eur Heart J. 2020, 2020. https://doi.org/10.1093/eurheartj/ehaa605. This paper represents the first sports cardiology Guidelines published by the ESC. It aims to aid the physicians when prescribing exercise programmes or recommendations for sports participation to athletes or individuals with cardiovascular diseases.

8. Sans S. Mediterranean diet, active lifestyle and cardiovascular disease: a recipe for immortality? Eur J Prev Cardiol. 2018;25:1182-5.

9. COVID-19 pulse: delivering weekly insights on the pandemic from a 150,000+ person connected cohort. [(accessed on 27 April 2020)]; Available online:http://web.archive.org/web/ 20200417084115/https://evidation.com/news/covid-19-pulse-firstdata-evidation/.

10. Stanton R, To QG, Khalesi S, Williams SL, Alley SJ, Thwaite TL, et al. Depression, Anxiety and stress during COVID-19: associations with changes in physical activity, sleep, tobacco and alcohol use in Australian adults. Int J Environ Res Public Health. 2020 Jun 7;17(11):4065. https://doi.org/10.3390/ijerph17114065.

11. Gallo LA, Gallo TF, Young SL, Moritz KM, Akison LK. The impact of isolation measures due To COVID-19 on Energy intake and physical activity levels in Australian university students. Nutrients 12, (2020);12(6):1865.

12. Górnicka M, Drywień ME, Zielinska MA, Hamułka J. Dietary and lifestyle changes during COVID-19 and the subsequent lockdowns among Polish adults: a cross-sectional online survey PLifeCOVID19 study. Nutrients 12, (2020);12(8):2324. https://doi.org/10.3390/ nu12082324

13. Sport England. New exercise habits forming during coronavirus crisis. London: Sport England; 2020.

14. Ding D, Cheng M, del Pozo Cruz B, Lin T, Sun S, Zhang L, et al. How COVID-19 lockdown and reopening affected daily steps: evidence based on 164,630 person-days of prospectively collected data from Shanghai, China. Int J Behav Nutr Phys Act. 2021;18:40.

15. Fukushima N, et al. Associations of working from home with occupational physical activity and sedentary behavior under the COVID-19 pandemic. J Occup Health. 2021;63:e12212.

16. Dunton GF, Do B, Wang SD. Early effects of the COVID-19 pandemic on physical activity and sedentary behavior in children living in the U.S. BMC Public Health. 2020;20:1351.

17. Schmidt SCE, Anedda B, Burchartz A, Eichsteller A, Kolb S, Nigg $\mathrm{C}$, et al. Physical activity and screen time of children and adolescents before and during the COVID-19 lockdown in Germany: a natural experiment. Sci Rep. 2020;10:21780.

18. Barkley JE, Lepp A, Glickman E, Farnell G, Beiting J, Wiet R, et al. The acute effects of the COVID-19 pandemic on physical activity and sedentary behavior in university students and employees. Int $\mathrm{J}$ Exerc Sci. 2020;13:1326-39.

19. Lesser IA, Nienhuis CP. The impact of COVID-19 on physical activity behavior and well-being of Canadians. Int J Environ Res Public Health. 2020 May 31;17(11):3899. https://doi.org/10.3390/ ijerph17113899.

20. Constandt B, Thibaut E, de Bosscher V, Scheerder J, Ricour M, Willem A. Exercising in times of lockdown: an analysis of the impact of COVID-19 on Levels and patterns of exercise among adults in Belgium. Int J Environ Res Public Health. 2020;17(11): 4144.

21. Meyer J, McDowell C, Lansing J, Brower C, Smith L, Tully M, et al. Changes in physical activity and sedentary behavior in response to COVID-19 and their associations with mental health in 3052 US adults. Int J Environ Res Public Health. 2020 Sep 5;17(18):6469. https://doi.org/10.3390/ijerph17186469.

22. Abate Daga F, Agostino S, Peretti S, Beratto L. COVID-19 nationwide lockdown and physical activity profiles among north-western Italian population using the International Physical Activity Questionnaire (IPAQ). Sport Sci Health. 2021:1-6. https://doi.org/ 10.1007/s11332-021-00745-8.

23. Aegerter AM, Deforth M, Sjøgaard G, Johnston V, Volken T, Luomajoki $\mathrm{H}$, et al. No evidence for a decrease in physical activity among Swiss office workers during COVID-19: a longitudinal study. Front Psychol. 2021;12:620307.

24. Tison GH, Avram R, Kuhar P, Abreau S, Marcus GM, Pletcher MJ, et al. Worldwide effect of COVID-19 on physical activity: a descriptive study. Ann Intern Med. 2020;173:767-70.

25. Amini H, Habibi S, Islamoglu AH, Isanejad E, Uz C, Daniyari H. COVID-19 pandemic-induced physical inactivity: the necessity of updating the global action plan on physical activity 2018-2030. Environ Health Prev Med. 2021;26:32.

26. Castelletti S. Dietary components and risk of cardiovascular disease and all-cause mortality: a review under the sign of the carrot. Eur J Prev Cardiol. 2019;26:1412-4.

27. Ammar A, Brach M, Trabelsi K, Chtourou H, Boukhris O, Masmoudi L, et al. Effects of COVID-19 Home confinement on eating behaviour and physical activity: results of the ECLBCOVID19 International Online Survey. Nutrients. 2020 May 28;12(6):1583. https://doi.org/10.3390/nu12061583.

28. Zupo R, Castellana F, Sardone R, Sila A, Giagulli VA, Triggiani V, et al. Preliminary Trajectories in dietary behaviors during the COVID-19 pandemic: a public health call to action to face obesity. Int J Environ Res Public Health. 2020 Sep 27;17(19):7073. https:// doi.org/10.3390/ijerph17197073.

29. Di Renzo L, et al. Eating habits and lifestyle changes during COVID-19 lockdown: an Italian survey. J Transl Med. 2020;18: 229.

30. Sánchez-Sánchez E, Ramírez-Vargas G, Avellaneda-López Y, Orellana-Pecino JI, García-Marín E, Díaz-Jimenez J. Eating habits and physical activity of the Spanish population during the COVID19 pandemic period. Nutrients. 2020;12(9):2826.

31. Reyes-Olavarría D, Latorre-Román PÁ, Guzmán-Guzmán IP, Jerez-Mayorga D, Caamaño-Navarrete F, Delgado-Floody P. Positive and negative changes in food habits, physical activity patterns, and weight status during COVID-19 confinement: associated factors in the chilean population. Int J Environ Res Public Health. 2020 Jul 28;17(15):5431. https://doi.org/10.3390/ijerph17155431.

32. Aldujeli A, Hamadeh A, Briedis K, Tecson KM, Rutland J, Krivickas Z, et al. Delays in presentation in patients with acute myocardial infarction during the COVID-19 pandemic. Cardiol Res. 2020;11:386-91.

33. De Rosa S, et al. Reduction of hospitalizations for myocardial infarction in Italy in the COVID-19 era. Eur Heart J. 2020;41:2083-8.

34. Garcia S, Albaghdadi MS, Meraj PM, Schmidt C, Garberich R, Jaffer FA, et al. Reduction in ST-segment elevation cardiac catheterization laboratory activations in the United States during COVID-19 pandemic. J Am Coll Cardiol. 2020;75:2871-2.

35. Negreira Caamaño M, Piqueras Flores J, Mateo Gómez C. Impact of COVID-19 pandemic in cardiology admissions. Med Clin (Barc). 2020;155:179-80.

36. Bollmann A, Pellissier V, Hohenstein S, König S, Ueberham L, Meier-Hellmann A, et al. Cumulative hospitalization deficit for cardiovascular disorders in Germany during the Covid-19 pandemic. Eur Heart J Qual Care Clin Outcomes. 2020. https://doi.org/10. 1093/ehjqcco/qcaa071.

37. Kiss P, Carcel C, Hockham C, Peters SAE. The impact of the COVID-19 pandemic on the care and management of patients with 
acute cardiovascular disease: a systematic review. Eur Heart J Qual Care Clin Outcomes. 2021;7:18-27.

38. Baldi E, Sechi GM, Mare C, Canevari F, Brancaglione A, Primi R, et al. Treatment of out-of-hospital cardiac arrest in the COVID-19 era: a 100 days experience from the Lombardy region. PLoS One. 2020;15:e0241028.

39. Marzolini S, de M Ghisi GL, Hébert A-A, Ahden S, Oh P. Cardiac rehabilitation in Canada during COVID-19. CJC Open. 2021;3: $152-8$.

40. Ko DT, Khera R, Lau G, Qiu F, Wang Y, Austin PC, et al. Readmission and mortality after hospitalization for myocardial infarction and heart failure. J Am Coll Cardiol. 2020;75:736-46.

41. Ribeiro F, Santos M. Exercise-based cardiac rehabilitation in COVID-19 times: one small step for health care systems, one giant leap for patients. Rev Esp Cardiol (Engl Ed). 2020;73:969-70. https://doi.org/10.1016/j.rec.2020.07.002.

42. Leon AS, Franklin BA, Costa F, Balady GJ, Berra KA, Stewart KJ, et al. Cardiac rehabilitation and secondary prevention of coronary heart disease: an American Heart Association scientific statement from the Council on Clinical Cardiology (Subcommittee on Exercise, Cardiac Rehabilitation, and Prevention) and the Council on Nutrition, Physical Activity, and Metabolism (Subcommittee on Physical Activity), in collaboration with the American Association Of Cardiovascular and Pulmonary Rehabilitation. Circulation. 2005;111:369-76.

43. Piepoli MF, Hoes AW, Agewall S, Albus C, Brotons C, Catapano $\mathrm{AL}$, et al. 2016 European guidelines on cardiovascular disease prevention in clinical practice: the Sixth Joint Task Force of the European Society of Cardiology and other societies on cardiovascular disease prevention in clinical practice (constituted by representatives of 10 societies and by invited experts)Developed with the special contribution of the European Association for Cardiovascular Prevention \& Rehabilitation (EACPR). Eur Heart J. 2016;37:2315-81.

44. Epstein E, Patel N, Maysent K, Taub PR. Cardiac rehab in the COVID era and beyond: mHealth and other novel opportunities. Curr Cardiol Rep. 2021;23:42.

45. Imran M, Wang L, McCrohon J, Yu C, Holloway C, Otton J, et al. Native T1 mapping in the diagnosis of cardiac allograft rejection: a prospective histologically validated study. JACC Cardiovasc Imaging. 2019;12:1618-28. https://doi.org/10.1016/j.jcmg.2018. 10.027 .

46. Thomas RJ, Beatty AL, Beckie TM, Brewer LC, Brown TM, Forman DE, et al. Home-Based cardiac rehabilitation: a scientific statement from the American Association of Cardiovascular and Pulmonary Rehabilitation, the American Heart Association, and the American College of Cardiology. Circulation. 2019;140:e6989.

47. Batalik L, Konecny V, Dosbaba F, Vlazna D, Brat K. Cardiac rehabilitation based on the walking test and telerehabilitation improved cardiorespiratory fitness in people diagnosed with coronary heart disease during the COVID-19 pandemic. Int J Environ Res
Public Health. 2021 Feb 24;18(5):2241. https://doi.org/10.3390/ ijerph18052241.

48.• Frederix I, et al. ESC e-Cardiology Working Group Position Paper: overcoming challenges in digital health implementation in cardiovascular medicine. Eur J Prev Cardiol. 2019;26:1166-77. This is the first ESC position paper aiming to provide a brief overview of currently existing the e-health applications. It describes their most relevant challenges and suggests how to overcome these challenges.

49. Mureddu GF, et al. Cardiac rehabilitation activities during the COVID-19 pandemic in Italy. Position Paper of the AICPR (Italian Association of Clinical Cardiology, Prevention and Rehabilitation). Monaldi Arch Chest Dis. 2020 Jun 15;90(2). https://doi.org/10.4081/monaldi.2020.1439.

50. Muriel B. Réadaptation cardiaque en période épidémique de COVID-19: propositions du GERS-P le 06/05/2020: Société Francaise de Cardiologie; 2020.

51. Kemps HMC, et al. Recommendations on how to provide cardiac rehabilitation services during the COVID-19 pandemic. Neth Heart J. 2020;28(7-8):387-390. https://doi.org/10.1007/s12471-020$01474-2$.

52. Vincente A, Raquel C. Applicability of the consensus of experts from heart failure/cardiac rehabilitation units and RehaCtivAP regarding the COVID-19. Rev Esp Cardiol Supl. 2020;20:22-4.

53. Ambrosetti M, Abreu A, Cornelissen V, Hansen D, Iliou MC, Kemps H, et al. Delphi consensus recommendations on how to provide cardiovascular rehabilitation in the COVID-19 era. Eur J Prev Cardiol. 2020;28:541-57. https://doi.org/10.1093/eurjpc/ zwaa080.

54. Banerjee A, Chen S, Pasea L, Lai AG, Katsoulis M, Denaxas S, et al. Excess deaths in people with cardiovascular diseases during the COVID-19 pandemic. Eur J Prev Cardiol. 2021. https://doi.org/ 10.1093/eurjpc/zwaa155.

55. Qian L, Ji KT, Nan JL, Lu Q, Zhu YJ, Wang LP, et al. Factors associated with decision time for patients with ST-segment elevation acute myocardial infarction. J Zhejiang Univ Sci B. 2013;14: 754-8.

56. Guerchicoff A, Brener SJ, Maehara A, Witzenbichler B, Fahy M, $\mathrm{Xu} \mathrm{K}$, et al. Impact of delay to reperfusion on reperfusion success, infarct size, and clinical outcomes in patients with ST-segment elevation myocardial infarction: the INFUSE-AMI Trial (INFUSEAnterior Myocardial Infarction). JACC Cardiovasc Interv. 2014;7: 733-40.

57. Tam C-CF, et al. Impact of coronavirus disease 2019 (COVID-19) outbreak on ST-segment-elevation myocardial infarction care in Hong Kong, China. Circ Cardiovasc Qual Outcomes. 2020;13: e006631.

Publisher's Note Springer Nature remains neutral with regard to jurisdictional claims in published maps and institutional affiliations. 